\title{
Glueball enhancement by color deconfinement
}

\author{
Vicente Vento* \\ Departamento de Física Teórica and Instituto de Física Corpuscular, Universidad de Valencia-Consejo Superior de Investigaciones \\ Científicas, 46100 Burjassot (València), Spain \\ (Received 21 September 2006; revised manuscript received 13 February 2007; published 21 March 2007)
}

\begin{abstract}
We study a scenario for high energy heavy ion collisions that leads to the formation of a strong coupling deconfined phase in which the lightest glueballs are numerous and stable. We analyze how their properties manifest themselves in experimental spectra and show that they provide a good signature for color deconfinement.
\end{abstract}

DOI: 10.1103/PhysRevD.75.055012

PACS numbers: $14.80 .-\mathrm{j}, 24.80 .+\mathrm{y}, 25.75 . \mathrm{Nq}$

\section{INTRODUCTION}

Quantum chromodynamics (QCD) is the theory of the strong interactions [1]. At low temperatures its elementary constituents are mesons, baryons and glueballs [2], this is the hadronic phase where all states are color singlets. At very high temperatures one expects a phase transition, called deconfinement, to take place. The new phase was thought to be a plasma of quarks and gluons and was named the quark gluon plasma (QGP) [3]. However, a recent formulation of the dynamics in the region above the transition temperature $T_{C}$ by Shuryak and Zahed, based on a description of recent experiments in ultrarelativistic heavy ion collisions [4], states that, despite deconfinement, the color Coulomb interaction between the constituents is strong and a large number of binary (even color) bound states, with a specific mass pattern, are formed [5]. This phase I call strong coulomb phase (SCP). The QGP phase occurs at a much higher temperature $T_{\mathrm{QGP}}>(2-3) T_{C}$ when the bound states disolve. I will label this realization of the phase transition as the SZ scenario. This description is not universally accepted since some lattice calculations do not to find bound states above the transition [6]. Also, one must be aware that other explanations of the data have been presented [7]. In order to clarify these issues it is interesting to find clear experimental observables that would shed some light into the discussion.

My aim is to study the behavior of QCD in the transition from the deconfined phase to the hadronic phase centering my attention in the behavior of the scalar glueballs. I will restrict my discussion to the SZ sceanrio although the analysis is more general. Other authors have proposed the glueballs to characterize deconfinement [8,9] but I single out in here a different dynamical picture.

Glueballs are bound states of gluons, the gauge bosons of QCD. This unique structure led to an intense experimental search, since they were first theoretically contemplated [2], which has not produced a clear picture of their spectrum. Moreover, they are expected to be broad because

*Email: Vicente.Vento@uv.es they mix strongly with quark states. The lightest glueball is a $0^{++}$, which I shall label by $g$.

Recently, I have proposed an interpretation of the scalar particles that contemplates a rich low lying glueball spectrum [10]. The analysis has been modeled by $\frac{1}{N_{C}}$ physics on which I have also based my estimates. I was led to a dynamical scenario where the OZI rule is broken and a low mass glueball, $g$, arises from the mixing of a pure OZI conserving glueball and a $\sigma$-meson, which are almost degenerate in mass. In this scenario $g$ is narrow although it remains hidden in the tale of the $\sigma$-meson. Experimentally they appear as a unique and broad resonance, the $f_{0}(600)$ [10].

My purpose is to show that these two states, $g$ and $\sigma$, behave in a very characteristic way across the deconfinement transition and therefore lead to observable effects associated with the SCP phase transition in the SZ scenario. My findings apply to any scenario for the phase transition that leads to an increase of the number of channels that decay into $g$.

\section{BEHAVIOR OF THE $\boldsymbol{g}$ WITH TEMPERATURE}

The realization of scale symmetry in gluodynamics (GD), the theory with gluons and no quarks, provides a relation between the parameters of the lightest scalar glueball, hereafter called $g$, and the gluon condensate [11-13],

$$
m_{g}^{2} f_{g}^{2}=-4\left\langle 0\left|\frac{\beta\left(\alpha_{s}\right)}{4 \alpha_{s}} G^{2}\right| 0\right\rangle,
$$

where $f_{g}=\langle 0|g| 0\rangle, m_{g}$ the $g$ mass, and the right hand side arises from the scale anomaly. GD provides a description for glueballs which almost coincides with that of QCD in the limit when the OZI rule is exactly obeyed, i.e., when decays into quarks which require gluons are strictly forbidden [10].

Lattice results $[14,15]$ and model calculations [16-18] support the traditional scenario $[19,20]$, that the condensate is basically constant up to the phase transition temperature $T_{C}\left(150 \mathrm{MeV}<T_{C}<300 \mathrm{MeV}\right)$ and decreases slowly thereafter until it dilutes (or evaporates) into gluons at (23) $T_{C}$. In this regime the mass of $g$ changes slowly across 

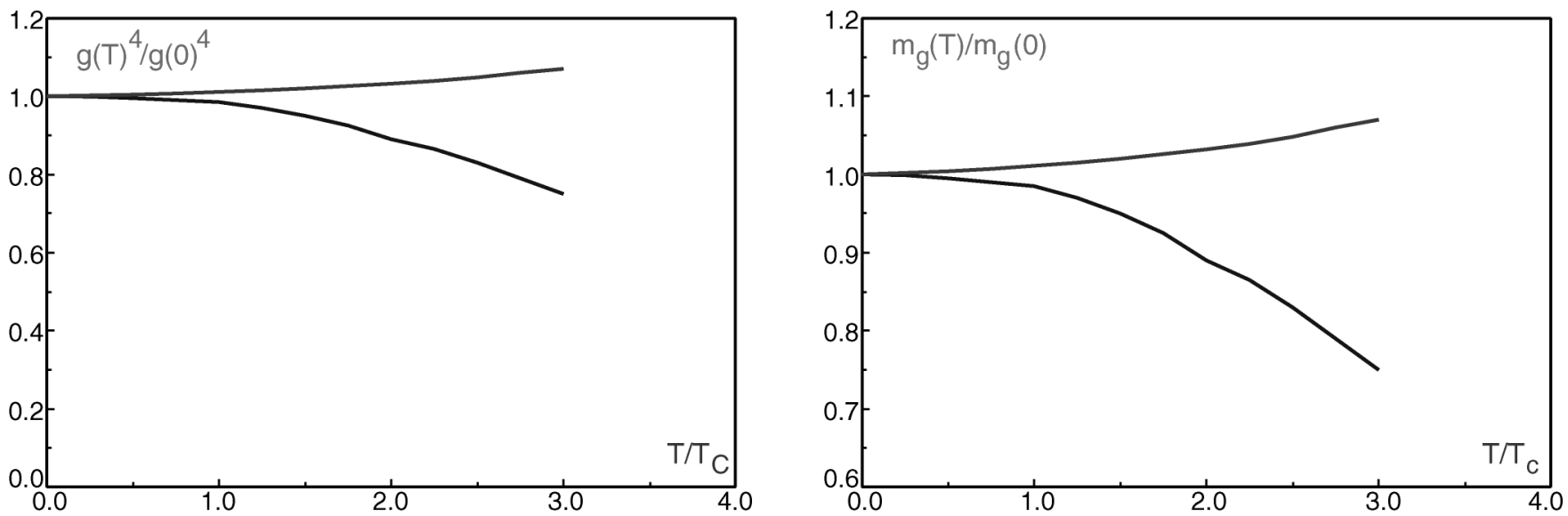

FIG. 1. Behavior of the gluon condensate, $g^{4}=m_{g}^{2} f_{g}^{2}$, and the mass, $m_{g}$, of $g$ across the deconfinement phase transition for various calculations mentioned in the text. The allowed values for different model calculations occur between the two lines.

the phase transition [16-18] and might even increase beyond $T_{C}$ as the gluon binding energy decreases [5] (see Fig. 1). These results and Eq. (1) determine that $f_{g}$ will be small only close to the dilution temperature when, in GD, scale invariance is restored. However, around $T_{C}, f_{g}$ is sizeable and therefore we are able to use in the scalar sector the OZI approximation of QCD, where glueballs and mesons are almost decoupled, and therefore the scalar glueballs of QCD behave similarly to those of GD [10].

I assume for the present discussion a recent formulation of the dynamics in the region above $T_{C}$, the $\mathrm{SZ}$ scenario, which states that despite deconfinement the color Coulomb interaction between the constituents is strong and a large number of binary (even color) bound states, with a specific mass pattern, are formed [5]. With this input, the scenario I envisage for GD goes as follows [21]. The strong Coulomb phase is crowded with gluon bound states and $g$ is the lightest. As one moves towards the dilution limit, the binding energy of these states decreases, the gluon mass increases, and therefore the color and singlet bound states increase their mass softly until the gluons are liberated forming a liquid $[5,22,23]$. However, as one cools towards the confining phase, color and singlet states decay into the conventional low lying glueballs, in particular $g$. Thus the number of $g$ 's becomes large.

Moreover, going to $\mathrm{QCD}$, one realizes that above the phase transition the multiplicity of glueball channels is larger than below. The ratio of glueball to meson channels goes from 1 to 8 below the phase transition to 1 to 2 above [5].

Thus our first important result is that the number of scalar glueballs is much larger in SCP than in the cold world.

The physical $g$ and $\sigma$ arise from the degenerate ones via mixing,

$$
g=g_{0} \cos (\theta / 2)-\sigma_{0} \sin (\theta / 2)
$$

$$
\sigma=g_{0} \sin (\theta / 2)+\sigma_{0} \cos (\theta / 2)
$$

where, the physical fields are expressed in terms of the degenerate OZI fields, $g_{0}$ and $\sigma_{0}$, and the mixing angle $\theta$.

The $\sigma$ meson decays into two pions or two photons [10],

$$
\begin{gathered}
\Gamma_{\sigma \rightarrow 2 \pi} \sim 1.5 \cos ^{2}(\theta / 2)\left(\frac{m_{\sigma}(\mathrm{GeV})}{1 \mathrm{GeV}}\right)^{3} \mathrm{GeV}, \\
\Gamma_{\sigma \rightarrow 2 \gamma} \sim 10.5 \cos ^{2}(\theta / 2)\left(\frac{m_{\sigma}(\mathrm{GeV})}{1 \mathrm{GeV}}\right)^{3} \mathrm{eV} .
\end{gathered}
$$

Therefore the physical scalar glueball $g$ decays, due to its $\sigma$ component, decays also into two pions or two photons [10],

$$
\begin{gathered}
\Gamma_{g \rightarrow 2 \pi} \sim 1.5 \sin ^{2}(\theta / 2)\left(\frac{m_{g}(\mathrm{GeV})}{1 \mathrm{GeV}}\right)^{3} \mathrm{GeV}, \\
\Gamma_{g \rightarrow 2 \gamma} \sim 10.5 \sin ^{2}(\theta / 2)\left(\frac{m_{g}(\mathrm{GeV})}{1 \mathrm{GeV}}\right)^{3} \mathrm{eV} .
\end{gathered}
$$

If we assume that the $\sigma$ is the $\mathrm{O}(4)$ partner of the $\pi$ in the chiral symmetry realization of QCD, its mass decreases when approaching the phase transition, becoming degenerate with the pion at $T_{C}$ (see Fig. 2). Beyond $T_{C}$, in the $\mathrm{SCP}$, chiral symmetry is restored, and $\pi$ and $\sigma$ remain degenerate for $T>T_{C}$. Thus in the SCP the $\sigma$ can only decay in $2 \gamma$ for obvious kinematical reasons. The glueball $g$ does not vary its mass in this region appreciably. Thus even before we reach $T_{C}$, the mixing between $g$ and $\sigma$ disappears (see Fig. 2) and $g$ becomes stable around $T_{C}$. However, in the SCP the mass of the $\sigma$ increases and in a certain region of $T$ it again becomes degenerate with $g$ and mixing is restored. Thus the physical $g$ is able to decay, once the $\sigma$ component is attained, to $2 \gamma$. 


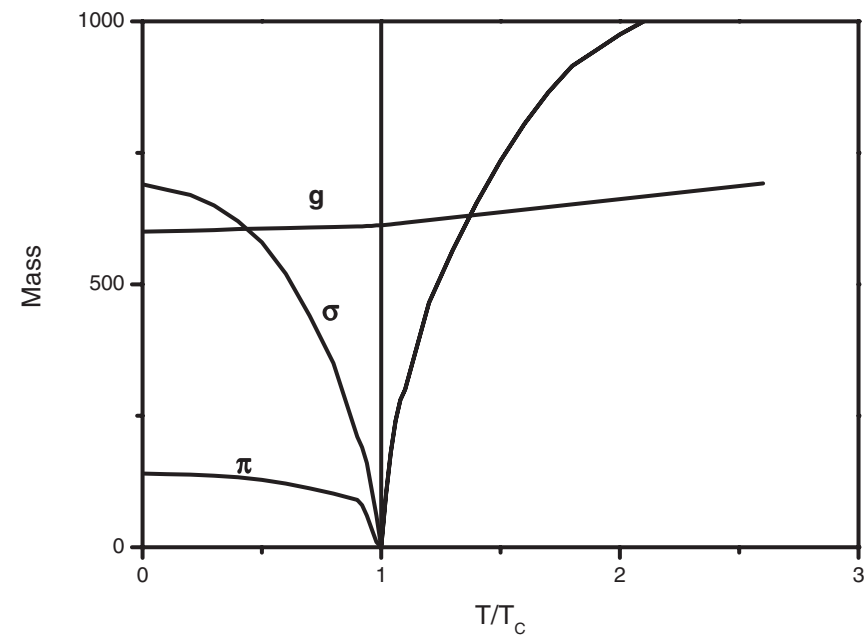

FIG. 2. Behavior of the masses of $\sigma, \pi$, and $g$ across the QGP phase transition according to model calculations.

Summarizing, in the SCP the ratio of the scalar glueballs to mesons increases and both the $g$ and the $\sigma$ can only decay to $2 \gamma$.

\section{THE COOLING OF THE FIREBALL}

When two heavy ions collide at ultrarelativistic energies, if the collision is quite central, a hot region of space time is produced called the fireball [4]. Let me incorporate in the cooling of the fireball the dynamics of QCD as described in the SZ scenario [5,21]. My starting point is SCP with a temperature $T_{C}<T<3 T_{C}$. This plasma, in this description, is almost a perfect fluid of hadronic matter with low viscosity and full of binary states [5]. The lowest mass $q \bar{q}$ states are the pseudoscalar pion $\pi$ and the scalar meson $\sigma$, which are here bound states of the strong color interaction. The lightest glueball state is $g$. The behavior of $g$ runs

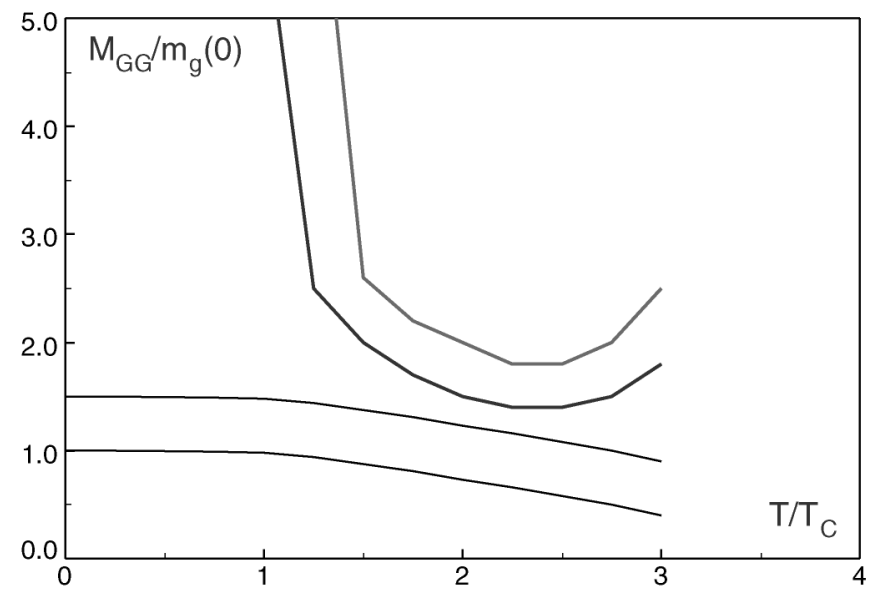

FIG. 3. Behavior of the glueball masses as one approaches the deconfinement phase transition. The mass of the color singlet states remains basically constant while the mass of the color states increases dramatically. together with all other hadronic processes leading to a collective flow but, in the OZI approximation, it can be singled out. Let me consider how the cooling of this plasma affects the population of glueballs and their general flow.

As the fireball cools a "large number" of gluonic bound states decay by gluon emission into $g$ 's. The emitted gluons form new bound states of lower mass due to the strong color Coulomb interaction. As we approach the confinement region the mass of the color bound states increases and it pays off to make multiparticle color singlet states, which decay by rearrangement into ordinary color singlet states (see Fig. 3). Since the coupling is strong and the phase space is large, these processes take place rapidly. Thus in no time, close to the phase transition temperature $T_{C}$, a large number of scalar glueballs populate the hadronic liquid. In our idealized OZI world they interact among themselves and with quark matter only by multi gluon exchanges, i.e., weak long range color Van der Waals forces. These forces allow the glueballs to be dragged by the hadronic liquid with the flow determined by the kinetics of the binary states from which they all proceed $[5,21]$.

\section{EXPERIMENTAL SIGNATURES}

I have presented all the ingredients necessary to discuss the observational signatures. I foresee two types of signatures: $2 \gamma$ decays and $2 \pi$ decays.

It is clear that the $\sigma$ and the $g$ will decay in the SCP only to $2 \gamma$. The width of the $\sigma$ will be strongly temperature dependent, due to the temperature dependence of its mass. On the contrary the $g$ will only decay in the temperature range where mixing takes place, i.e. when their masses are close (see Fig. 2). Besides their mass variation both widths will be broaden by the energy of the heat bath. Thus we expect a broad $\sigma$ meson, whose width is temperature dependent, and a narrow $g$ whose width is temperature independent but has a temperature threshold. The enhancement in the $g$ with respect to the hadronic phase arises because of the larger population in the SCP as described above, since the ratio of glueball channels to meson channels changes from 1:8 to $1: 2$, and because these particles are stable in the medium against the dominating hadronic decays. In order to arrive from the zero temperature elementary processes as described by Eqs. (5) and (7), see Ref. [10]) for details, to the experimental data one has to perform a complicated calculation taking into account temperature dependences and evolution, which is outside the scope of this presentation. However, our experimental colleagues have performed a beautiful analysis eliminating the background and have obtained an beautiful isolated signal for the $\pi_{0} \rightarrow 2 \gamma$ case [24]. I expect that due to the similar behavior of our states in the heat bath (see Fig. 2), an analogous result will be obtained for the $\sigma$, while the $g$ will behave also similarly except for the increase in population discussed above. In order to obtain plausible expec- 

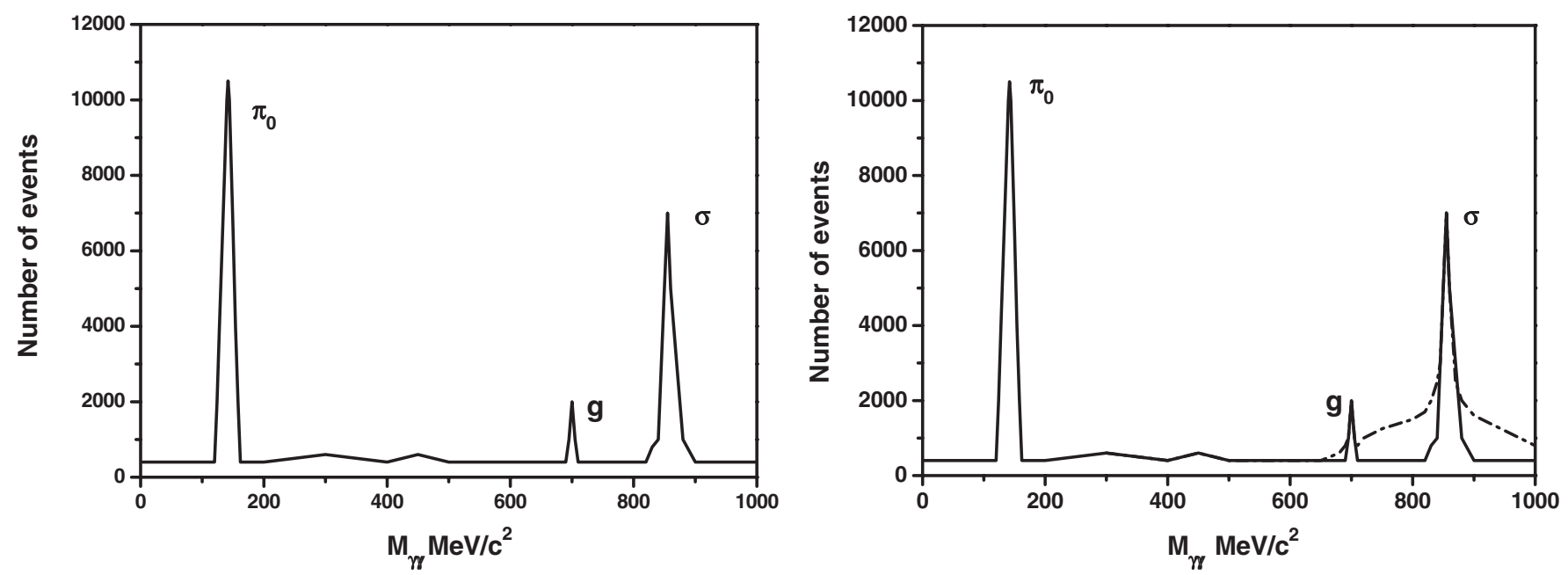

FIG. 4. Expected fit to the two-photon invariant mass spectrum in central $\mathrm{Pb}-\mathrm{Pb}$ collisions after substraction from the background. The $2 \gamma$ decays should allow for a clear separation of $g$ and $\sigma$. The left figure shows the pure electromagnetic calculation, which dominates de SCP, while in the right figure I estimate the effect of the hadronic widths on the fits (dashed-dotted line).

tations, Fig. 4, I have scaled the zero temperature results for $\sigma$ and $g$ as in the pion, while taking also the increase in population for the $g$ into account. If our expectations are correct the PHOS detector at LHC, whose capability of detecting $\pi_{0}$ is impressive, should be able to detect both the $\sigma$ and the $g$ at slightly higher kinematics than shown in Ref. [24]. ${ }^{1}$

Let me now look into $2 \pi$ decays in the medium, i.e., into the hadronic widths of $\sigma$ and $g$ (recall Eqs. (4) and (6) and Ref. [10] for details). $\sigma$ and $g$ are stable against these type of decays until $m_{\sigma}$ becomes greater than $2 m_{\pi}$ for the former, and mixing takes place for the latter. Thus they cross the phase transition temperature, $T_{C}$, as stable states (see Fig. 2). This mechanism provides us with a "time delay" associated with the cooling after the phase transition [21]. The delay will appear as a narrowing of the hadronic widths. One further effect which enhances the signal is the increase in the number of events due to the larger population of glueballs in the SCP. Thus the count rate for the $g$ will increase while the widths of both peaks will narrow. Unluckily, these decays seem, at present, undetectable in ultrarelativistic heavy ion reactions due to the huge number of pions coming out of the fireball. However, we can conclude, for later use, that the hadronic widths of the $\sigma$ and $g$ become smaller.

Let us turn this argument around to rediscuss the $2 \gamma$ decays. In our previous calculation we have not taken into account the hadronic width of these particles. Certainly they will affect the $2 \gamma$ process as they do affect the dilepton spectrum in the decay of the vector mesons [25]. The fact that $g$ and $\sigma$ do not decay hadronically in the SCP, they

\footnotetext{
${ }^{1}$ I expect that the broadening of the $\pi_{0}$ width is not only due to the heat bath but also to the temperature dependence of the pion mass.
}

only do below $T_{C}$, that their widths become narrower and that the rates of $g$ higher, give the glueball a chance to come out of the $\sigma$ background (see Fig. 4).

Let me conclude by stating that I have analyzed the behavior of a peculiar hadronic state, the scalar glueball, $g$, in a hot hadronic medium. This state, according to a recent description [10] appears in nature mixed with a scalar meson, $\sigma$. I have discussed in physical terms how these particles, which are created copiously in the strong color Coulomb phase, behave as the fireball cools down. We have seen that the weak coupling of $g$ with other hadronic states and the chiral properties of the $\sigma$ provide them with a well defined behavior in the plasma as the temperature drops. This behavior is transferred to its detectable decay products leading to a sizeable emission of photons which hints a possible signature for SCP formation. The large production of $g$ 's in the medium allows for enhanced counting rates compared with the zero temperature scenario. Finally, our discussion can be generalized to any scenario for the phase transition that increases the number of glueball states in comparison with the zero temperature theory.

\section{ACKNOWLEDGMENTS}

I would like to thank K. Langfeld and B.-J. Schaefer for clarifying information regarding their work. I acknowledge the hospitality extended to me by the GSI theory group, were this research was initiated, and, in particular, the conversations with Bengt Friman were illuminating. I thank Yu. Kharlov for providing me with his contribution prior to publication and additional references. I thank $\mathrm{C}$. Cioffi and D. Treleani for inviting me to the Trieste conference where this piece of work took shape. This work was supported by MCYT-FIS2004-05616-C02-01 and GVGRUPOS03/094. 
[1] H. Fritzsch, M. Gell-Mann, and H. Leutwyler, Phys. Lett. B 47, 365 (1973).

[2] H. Fritzsch and P. Minkowski, Nuovo Cimento Soc. Ital. Fis. A 30, 393 (1975).

[3] E. V. Shuryak, Phys. Rep. 61, 71 (1980).

[4] Nucl. Phys. A757 1 (2005), First three years of operation of RHIC.

[5] E. Shuryak and I. Zahed, Phys. Rev. D 69, 046005 (2004); E. V. Shuryak and I. Zahed Phys. Rev. C 70, 021901(R) (2004).

[6] F. Karsch, S. Ejiri, and K. Redlich, Nucl. Phys. A774, 619 (2006); V. Koch, A. Majumder, and J. Randrup, Phys. Rev. Lett. 95, 182301 (2005).

[7] C. Ratti, S. Rößner, M. A. Thaler, and W. Weise, hep-ph/ 0609218.

[8] E. V. Shuryak, Phys. Lett. B 515, 359 (2001).

[9] Nikolai Kochelev and Dong-Pil Min, hep-ph/0611250; Phys. Lett. B 633, 283 (2006); Phys. Rev. D 72, 097502 (2005).

[10] V. Vento, Phys. Rev. D 73, 054006 (2006).

[11] J. Schechter, Phys. Rev. D 21, 3393 (1980).

[12] A. A. Migdal and M. A. Shifman, Phys. Lett. B 114, 445 (1982).

[13] J. Lanik, Phys. Lett. B 144, 439 (1984); J. Ellis and J. Lanik, Phys. Lett. B 150, 289 (1985).

[14] T. Kodama, Braz. J. Phys. 34, 205 (2004); P. Petreczky, Nucl. Phys. B, Proc. Suppl. 140, 78 (2005); F. Karsch,
Lect. Notes Phys. 583, 209 (2002).

[15] K. Lanfeld et al., Nucl. Phys. B, Proc. Suppl. 106, 501 (2002).

[16] J. Sollfrank and U. Heinz, Z. Phys. C 65, 111 (1995).

[17] A. Drago, M. Gibilisco, and C. Ratti, Nucl. Phys. A742, 165 (2004).

[18] B.-J. Schaefer, O. Bohr, and J. Wambach, Phys. Rev. D 65 , 105008 (2002).

[19] H. Leutwyler, in Deconfinemente and Chiral Symmetry, edited by P.M. Zerwas and H.A. Castrup (World Scientific, Singapore, 1993).

[20] D. E. Miller, Acta Phys. Pol. B 28, 209 (2002); hep-ph/ 9807304.

[21] V. Vento, nucl-th/0509102.

[22] A. M. Polyakov, Phys. Lett. B 82, 247 (1979).

[23] A. Linde, Phys. Lett. B 96, 289 (1980).

[24] G. Conesa, M. Ippolitov, Yu. V. Kharlov, V. Manko, D. Peressounko, S. Sadovsky, and Y. Schutz, Contribution to the 5th International Conference on Perspectives in Hadronic Physics Particle-Nucleus and Nucleus-Nucleus Scattering at Relativistic Energies, Trieste, 2006, [Nucl. Phys. A (to be published)]; G. Conesa, H. Delagrange, J. Diaz, M. Ippolitov, Yu. V. Kharlov, D. Peressounko, and Y. Schutz, Nucl. Instrum. Methods Phys. Res., Sect. A 537, 363 (2005).

[25] S. Damjanovic, nucl-ex/0609026. 